\title{
Particle-in-cell simulations of parametric decay instabilities at the upper hybrid layer of fusion plasmas to determine their primary threshold
}

Senstius, Mads Givskov; Nielsen, Stefan Kragh; Vann, R. G.; Hansen, Søren Kjer

Published in:

Plasma Physics and Controlled Fusion

Link to article, DOI:

10.1088/1361-6587/ab49ca

Publication date:

2020

Document Version

Peer reviewed version

Link back to DTU Orbit

Citation (APA):

Senstius, M. G., Nielsen, S. K., Vann, R. G., \& Hansen, S. K. (2020). Particle-in-cell simulations of parametric decay instabilities at the upper hybrid layer of fusion plasmas to determine their primary threshold. Plasma Physics and Controlled Fusion, 62(2), [025010]. https://doi.org/10.1088/1361-6587/ab49ca

\section{General rights}

Copyright and moral rights for the publications made accessible in the public portal are retained by the authors and/or other copyright owners and it is a condition of accessing publications that users recognise and abide by the legal requirements associated with these rights.

- Users may download and print one copy of any publication from the public portal for the purpose of private study or research.

- You may not further distribute the material or use it for any profit-making activity or commercial gain

- You may freely distribute the URL identifying the publication in the public portal 
ACCEPTED MANUSCRIPT

\title{
Particle-in-cell simulations of parametric decay instabilities at the upper hybrid layer of fusion plasmas to determine their primary threshold
}

To cite this article before publication: Mads Givskov Senstius et al 2019 Plasma Phys. Control. Fusion in press https://doi.org/10.1088/1361$\underline{6587 / a b 49 c a}$

\author{
Manuscript version: Accepted Manuscript \\ Accepted Manuscript is "the version of the article accepted for publication including all changes made as a result of the peer review process, \\ and which may also include the addition to the article by IOP Publishing of a header, an article ID, a cover sheet and/or an 'Accepted \\ Manuscript' watermark, but excluding any other editing, typesetting or other changes made by IOP Publishing and/or its licensors" \\ This Accepted Manuscript is @ 2019 IOP Publishing Ltd.
}

During the embargo period (the 12 month period from the publication of the Version of Record of this article), the Accepted Manuscript is fully protected by copyright and cannot be reused or reposted elsewhere.

As the Version of Record of this article is going to be / has been published on a subscription basis, this Accepted Manuscript is available for reuse under a CC BY-NC-ND 3.0 licence after the 12 month embargo period.

After the embargo period, everyone is permitted to use copy and redistribute this article for non-commercial purposes only, provided that they adhere to all the terms of the licence https://creativecommons.org/licences/by-nc-nd/3.0

Although reasonable endeavours have been taken to obtain all necessary permissions from third parties to include their copyrighted content within this article, their full citation and copyright line may not be present in this Accepted Manuscript version. Before using any content from this article, please refer to the Version of Record on IOPscience once published for full citation and copyright details, as permissions will likely be required. All third party content is fully copyright protected, unless specifically stated otherwise in the figure caption in the Version of Record.

View the article online for updates and enhancements. 
Submitted to: Plasma Phys. Control. Fusion

\title{
Particle-in-cell simulations of parametric decay instabilities at the upper hybrid layer of fusion plasmas to determine their primary threshold
}

\author{
M G Senstius ${ }^{1}$, S K Nielsen ${ }^{1}$, R G Vann ${ }^{2}$, S K Hansen ${ }^{1,3}$
}

${ }^{1}$ Department of Physics, Technical University of Denmark, Fysikyej, DK-2800 Kgs. Lyngby, Denmark

${ }^{2}$ York Plasma Institute, Department of Physics, University of York, York YO10 5DD, United Kingdom

${ }^{3}$ Max-Planck-Institut für Plasmaphysik, Boltzmannstraße, D-85748 Garching bei München, Germany

\begin{abstract}
Parametric decay instabilities (PDIs) are nonlinear processes by which energy from a strong pump wave may be directed into other waves at other frequencies, in particular natural modes of the medium, provided that energy and momentum are conserved. The particle-in-cell (PIC) code EPOCH is used to simulate PDIs in a magnetically confined fusion plasma converting a $105 \mathrm{GHz}$ microwave $\mathrm{X}$-mode pump wave into electrostatic daughter waves at the upper hybrid (UH) layer. Modes associated with the PDIs as well as a linearly converted electron Bernstein wave (EBW) are identified in $f-$ and $k$-space. The PDI daughter modes are found to agree with experimental observations from ASDEX Upgrade as well as with analytical predictions, showing a nonlinear increase in power above a predicted threshold.
\end{abstract}

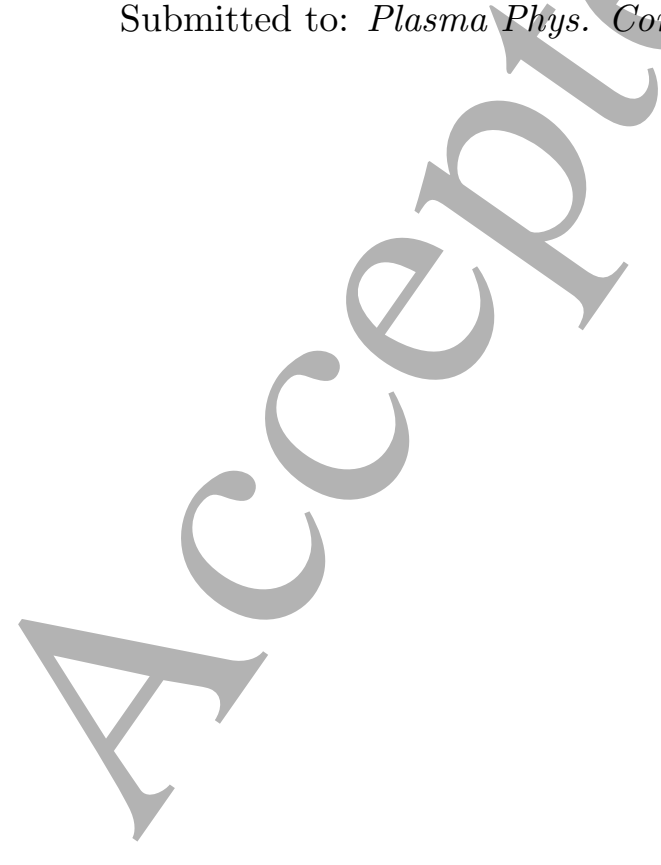




\section{PIC simulations of PDIs at the UH layer}

\section{Introduction}

Parametric decay instabilities[1, 2, 3, 4, 5, 6] (PDIs) are a group of fundamental nonlinear processes involving interactions and a transfer of energy between different waves. Any type of wave may take part provided that certain selection rules are satisfied and that an amplitude threshold is exceeded. Both mechanical oscillations and electromagnetic waves may undergo PDI[7, 8]. In plasmas, large amplitude waves have been known to undergo PDI in the ionosphere[9], in inertial confinement fusion experiments[10] as well as in magnetic confinement plasmas[11, 12, 13, 14, 15, 16, 17, 18, 19, 20, 21].

High power microwaves are important in the study of magnetically confined fusion plasmas due, in part, to the electron cyclotron (EC) frequency typically being in the microwave range. PDI driven by high power microwaves has been observed in a number of experiments related to electron cyclotron resonance heating (ECRH) and electron cyclotron current drive (ECCD) [12, 22]. Furthermore, PDI has been observed during electron Bernstein wave (EBW) heating[16] and scattering experiments such as collective Thomson scattering (CTS) [20]. The waves produced by PDI can potentially alter plasma behavior and damage first wall and diagnostic components. Additionally, PDI may divert energy into unintended waves, causing heating and current drive to take place in the wrong location of the plasma.

PDI involving externally launched microwave gyrotron radiation was experimentally first indicated at Versator II [12] and has recently been demonstrated consistently at ASDEX Upgrade [20, 21]. This special case/where an X-mode wave decays into two electrostatic daughter waves near the (UH) layer has received renewed attention by a number of authors[23, 24]. Based on observations in ASDEX Upgrade, Hansen et al[23] derived dispersion relations for the implicated waves in the PDI as well as a power threshold which is lowered due to wave amplification near the upper hybrid UH layer. The decay of trapped waves near UH layer is also a key element in determining the power threshold for the two plasmon decay instability in ECRH experiments[25, 26]. Although these analytical models yield fitting results, they are often based om a dipole approximation, i.e. the wavenumber of the pump is much smaller than that of the daughter waves. Under most conditions, the wavenumber of an X-mode pump wave is much smaller than the daughter waves, thus justifying the dipole approximation. However at the UH layer, the wavenumber of the X-mode wave is increased significantly, making it comparable to that of the electrostatic daughter waves. Furthermore, the analytical models require prior knowledge of the interacting waves.

Numerical codes allows for studies of PDI without the need for the assumptions applied in analytical models and can therefore be used to test the validity of the models. Particle-in-cell (PIC) code simulations of X-B conversion have shown indications of concurrent nonlinear conversion through a PDI[27]. In this article, PIC code simulations are set up to systematically investigate PDI near the UH layer of a magnetized plasma. The simulations allow for identification of implicated modes in terms of both frequency and wavenumber. With this information, the validity of the dipole approximation, often 
employed in analytical work, is assessed and PDI selection rules are checked to determine which waves interact. The dipole approximation is assessed dubious near the UH layer. Nevertheless, the analytically derived threshold for the PDI of an X-mode pump wave near the UH layer is still found to agree with numerical observations. Additionally, EBWs generated at the UH layer through linear conversion are found to also undergo PDI and a power threshold for this PDI is estimated.

\section{PDI theory}

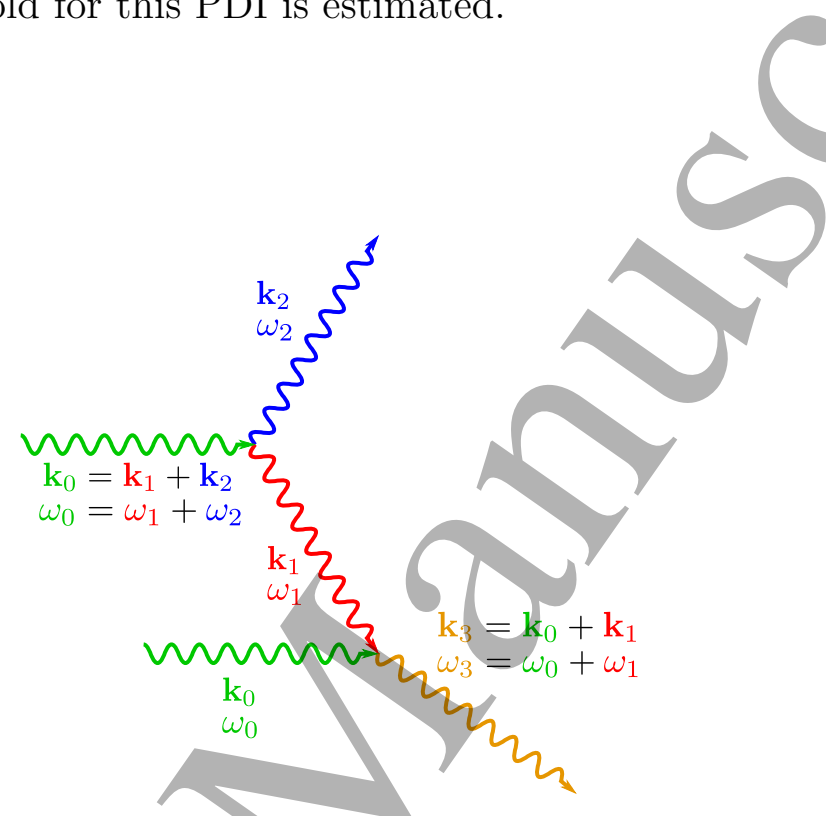

Figure 1. An idealized representation of parametric decay as described in equations (1a-b). A pump wave, $\mathbf{k}_{0}, \omega_{0}$, may decay into two daughter waves, $\mathbf{k}_{1}, \omega_{1}$ and $\mathbf{k}_{2}, \omega_{2}$, provided that the sum of wave vectors and of angular frequencies are conserved. One of the daughters may then recombine with the pump to produce another wave, $\mathbf{k}_{3}, \omega_{3}$.

The parametric decay processes of focus in the present article are three-wave interactions where an X-mode pump wave, denoted by its wave vector $\mathbf{k}_{0}$ and frequency $f_{0}$, near the UH frequency decays into a downshifted EBW, $\mathbf{k}_{2}$ and $f_{2}$, and a warm LH wave, $\mathbf{k}_{1}$ and $f_{1}$, at a much lower frequency. The warm LH daughter may then recombine with the pump wave to produce an upshifted EBW daughter, $\mathbf{k}_{3}$ and $f_{3}$. This is illustrated in figure 1. The rate at which these processes occur depends nonlinearly on the amplitudes of all the implicated waves. Above some threshold pump wave amplitude, the decay processes become unstable and only then will the daughter waves of the PDI grow above the thermal noise level. Conservation of energy and momentum leads to the following matching conditions for the two processes

$$
\begin{array}{ll}
\omega_{0}=\omega_{1}+\omega_{2}, & \mathbf{k}_{0}=\mathbf{k}_{1}+\mathbf{k}_{2}, \\
\omega_{3}=\omega_{0}+\omega_{1}, & \mathbf{k}_{3}=\mathbf{k}_{0}+\mathbf{k}_{1} .
\end{array}
$$

Where the angular frequencies, of course, relate to the frequencies by $\omega=2 \pi f$. In a homogeneous plasma, the matching conditions may be satisfied over a large physical extend, lowering the pump power threshold of a PDI $[4,5,6]$. In an inhomogeneous plasma, the matching conditions may only be satisfied in a very limited range of positions, thus increasing the threshold. Amplification of the pump wave at the UH 


\section{PIC simulations of PDIs at the UH layer}

layer, however, means that the power threshold of an X-mode beam propagating to the $\mathrm{UH}$ layer is lowered and growth rates can be greater. The $\mathbf{k}$ - and $f$-matching just have to be satisfied locally in an inhomogeneous plasma for the decay to takeplace.

The daughter waves investigated in this article are electrostatic waves with $\omega_{1} \approx$ $\omega_{\mathrm{LH}}$ and $\omega_{2} \approx \omega_{\mathrm{UH}}$, which means that $\omega_{1} \ll \omega_{2}$. An analytical expression for the frequency shifts is derived in [23] by employing a dipole approximation, i.e. $\mathbf{k}_{0} \approx \mathbf{0}$. Assuming that the propagation is perpendicular to the magnetic field, the frequency shift can be further reduced to

$$
\omega_{1} \approx \omega_{\mathrm{LH}}\left(1+\frac{A_{1}}{2} \frac{\omega_{\mathrm{UH}}^{2}-\omega_{0}^{2}+2 \omega_{0} \omega_{\mathrm{LH}}}{A_{2} \omega_{\mathrm{pe}}^{2}-A_{1} \omega_{0} \omega_{\mathrm{LH}}}\right)
$$

with the dimensionless parameters $A_{1}, A_{2}$ defined as

$$
\begin{aligned}
& A_{1}=3\left[\frac{\omega_{\mathrm{pe}}^{2}}{4 \omega_{\mathrm{UH}}^{2}}+\frac{T_{\mathrm{i}}}{Z_{\mathrm{i}} T_{\mathrm{e}}} \frac{\omega_{\mathrm{UH}}^{2}}{\omega_{\mathrm{pe}}^{2}}\right], \\
& A_{2}=\left(1-\frac{\omega_{\mathrm{pe}}^{2}}{3 \omega_{\mathrm{ce}}^{2}}\right)^{-1} .
\end{aligned}
$$

Here $\omega_{\mathrm{c} \sigma}=q_{\sigma} B / m_{\sigma}$ and $\omega_{\mathrm{p} \sigma}=\sqrt{n_{\sigma} q_{\sigma}^{2} /\left(\epsilon_{0} m_{\sigma}\right)}$ are the cyclotron and plasma angular frequency for particle $\sigma \in\{\mathrm{e}, \mathrm{i}\}$, where $\mathrm{e}$ is for electron and $\mathrm{i}$ is for ion. The thermal velocity and Larmor radius of particle species $\sigma$ are $v_{\mathrm{t} \sigma}=\sqrt{2 T_{\sigma} / m_{\sigma}}$ and $r_{\mathrm{L} \sigma}=$ $v_{\mathrm{t} \sigma} /\left(\sqrt{2}\left|\omega_{\mathrm{c} \sigma}\right|\right)$ respectively. The quantities $m_{\sigma}, n_{\sigma}$ and $T_{\sigma}$ are particle mass, density and temperature, and the angular frequencies $\omega_{\mathrm{UH}}=\sqrt{\omega_{\mathrm{ce}}^{2}+\omega_{\mathrm{pe}}^{2}}$ and $\omega_{\mathrm{LH}}=\left|\omega_{\mathrm{ce}} \omega_{\mathrm{pi}} / \omega_{\mathrm{UH}}\right|$ are the upper hybrid and lower hybrid angular frequency. The warm LH and downshifted EBW daughter waves are described by the dispersion relations

$$
\begin{aligned}
& k_{1}^{2} \approx\left(\frac{\omega_{1}^{2}}{\omega_{\mathrm{LH}}^{2}}-1\right) /\left(A_{1} r_{\mathrm{Le}}^{2}\right), \\
& k_{2}^{2} \approx \frac{\omega_{\mathrm{UH}}^{2}-\omega_{2}^{2}}{A_{2} r_{\mathrm{Le}}^{2} \omega_{\mathrm{pe}}^{2}},
\end{aligned}
$$

where $\omega_{2}=\omega_{0}-\omega_{1}$ following equation $(1 a)$.

The pump is here described by the warm X-mode dispersion relation[28], which for propagation perpendicular to the magnetic field becomes

$$
0=\left(1-\sum_{\sigma} S_{1 \sigma}\right)\left(1-\frac{k_{0}^{2} c^{2}}{\omega_{0}^{2}}-\sum_{\sigma} S_{2 \sigma}\right)-\left(\sum_{\sigma} S_{3 \sigma}\right)^{2},
$$

where

$$
\begin{aligned}
& S_{1 \sigma}=\frac{\omega_{\mathrm{p} \sigma}^{2} e^{-\lambda_{\sigma}}}{\omega_{0} \lambda_{\sigma}} \sum_{n=-\infty}^{\infty} \frac{n^{2} I_{n}\left(\lambda_{\sigma}\right)}{\omega_{0}-n \omega_{\mathrm{c} \sigma}} \\
& S_{2 \sigma}=\frac{\omega_{\mathrm{p} \sigma}^{2} e^{-\lambda_{\sigma}}}{\omega_{0} \lambda_{\sigma}} \sum_{n=-\infty}^{\infty} \frac{n^{2} I_{n}\left(\lambda_{\sigma}\right)+2 \lambda_{\sigma}^{2} I_{n}\left(\lambda_{\sigma}\right)-2 \lambda_{\sigma}^{2} I_{n}^{\prime}\left(\lambda_{\sigma}\right)}{\omega_{0}-n \omega_{\mathrm{c} \sigma}} \\
& S_{1 \sigma}=\frac{\omega_{\mathrm{p} \sigma}^{2} e^{-\lambda_{\sigma}}}{\omega_{0}} \sum_{n=-\infty}^{\infty} \frac{n\left[I_{n}^{\prime}\left(\lambda_{\sigma}\right)-I_{n}\left(\lambda_{\sigma}\right)\right]}{\omega_{0}-n \omega_{\mathrm{c} \sigma}}
\end{aligned}
$$


1

2

3

4

5

6

7

8
PIC simulations of PDIs at the UH layer

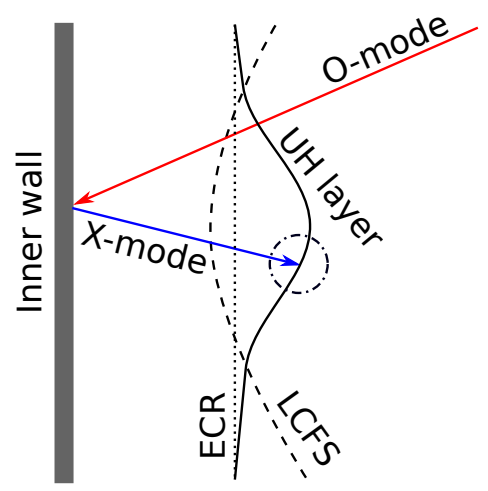

Figure 2. A sketch showing the typical placement of resonances and beam paths that lead to strong scattering in ASDEX Upgrade. The red arrow represents in incoming $\mathrm{O}$-mode gyrotron beam that is converted to X-mode at the inner wall. The X-mode, represented by a blue arrow, propagates back into the plasma, passing the last closed flux surface (LCFS), the electron cyclotron resonance (ECR) and reaches the upper hybrid (UH) layer. The interaction region where the scattering is believed to emanate from is marked by the dash-dotted line.

for $\lambda_{\sigma}=k_{0}^{2} r_{\mathrm{L} \sigma}^{2}$ and $\sigma \in\{\mathrm{e}, \mathrm{i}\} . I_{n}\left(\lambda_{\sigma}\right)$ is the $n$th modified Bessel function and $I_{n}^{\prime}\left(\lambda_{\sigma}\right)$ is its derivative. As the pump gets closer to the UH layer, it becomes increasingly electrostatic and eventually coincides with the first EBW branch. The pump wave can therefore be linearly converted into an EBW at the UH layer[16]. This EBW, unlike the frequency shifted EBW PDI daughter waves, is exactly at the pump frequency. The dispersion relation for EBWs is given by [28],

$$
0=1-\frac{\omega_{\mathrm{pe}}^{2} e^{-\lambda_{\mathrm{eL}}}}{\omega_{\mathrm{L}} \lambda_{\mathrm{eL}}} \sum_{n=-\infty}^{\infty} \frac{n^{2} I_{n}\left(\lambda_{\mathrm{eL}}\right)}{\omega_{\mathrm{L}}-n \omega_{\mathrm{ce}}}
$$

where $\lambda_{\mathrm{eL}}=k_{\mathrm{L}}^{2} r_{\mathrm{Le}}^{2}$, and $\omega_{\mathrm{L}}, k_{\mathrm{L}}$ are the angular frequency and wave number of the linearly converted EBW. The notable differences between this linearly converted EBW and the pump are that the EBW propagates much slower, i.e. $\left|v_{0} / v_{\mathrm{L}}\right| \ll 1$, and has a greater absolute wave number, i.e. $\left|k_{0} / k_{\mathrm{L}}\right| \ll 1$, when the waves are not at the UH layer. The pump and the linearly converted EBW propagate in different directions; in 1D, they propagate in opposite directions.

\section{Numerical Setup}

\subsection{Accessibility to the UH layer}

The simulations of this article are designed so that the parameters at the UH layer resemble that of the ASDEX Upgrade discharge described in [23]. Strong scattering was observed when an X-mode beam reached the UH layer. Getting an X-mode beam to the UH layer, however, is not straightforward as it is reflected at the R-cutoff when launched from the low field side. An O-mode beam launched from the low field side, which passes through the plasma, is reflected and partly converted to X-mode on the high field side inner wall. Before reaching the UH layer, the reflected X-mode passes 


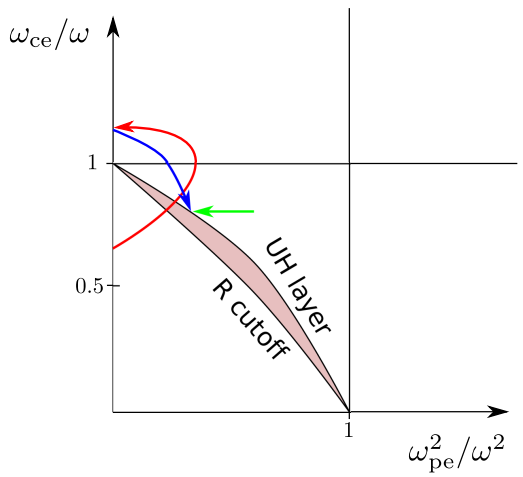

Figure 3. A simplified CMA diagram featuring the approximate beam path to reach the UH layer (red and blue arrows) in ASDEX Upgrade shot 28286 and the beam path to be used in simulations (green arrow). The red arrow/represents the path of an O-mode launched from the low field side. The blue arrow is the $\mathrm{X}$-mode that is generated from the O-mode at the high field side and reflected back into the plasma. The Green arrow represents the path of an X-mode beam generated in simulations.

the electron cyclotron resonance (ECR) where it is partly absorbed. A fraction of the $\mathrm{X}$-mode nevertheless makes it to the UH layer and scattering is then observed at approximately $f_{0} \pm f_{\mathrm{LH}}$. This trajectory is illustrated in figure 2 while figure 3 shows the path in a CMA diagram. Having reached the $\mathrm{UH}$ layer, the $\mathrm{X}$-mode is unable to propagate further and is then converted either through PDI or by a linear conversion into an EBW[26], which changes its direction of propagation. In this work, only an Xmode already near the UH layer is considered, i.e. the green arrow is followed to lower the computational costs. The reflection and conversion of O-mode will not discussed in further detail.

\subsection{Simulations}

The code used for the simulations is the second order relativistic PIC code EPOCH $[27$, 29]. Like any PIC code, physical particles are modeled as a smaller number of pseudoparticles that represent clouds of physical particals, adding up to the same properties as that of the desired plasma. The routine is a split step process between updating particle dynamics due to the electromagnetic fields and updating the electromagnetic fields due to the currents induced by the moving charged particles using a finite difference approach.

While EPOCH is capable of doing both 2D and 3D simulations, the simulations in this article were in 1D as all the important dynamics are expected to rely on changes in the direction of propagation, provided that this is perpendicular to the magnetic field. Although the simulations are 1D, EPOCH retains 3 dimensions on all vector quantities such as the electric field, the magnetic field and the velocity of the pseudoparticles. In only one inhomogeneous spatial dimension, a position dependent background magnetic field that is both stationary and divergence free is not possible. For this reason, the beam path of the experiment described earlier is not possible. Instead, the magnetic field 

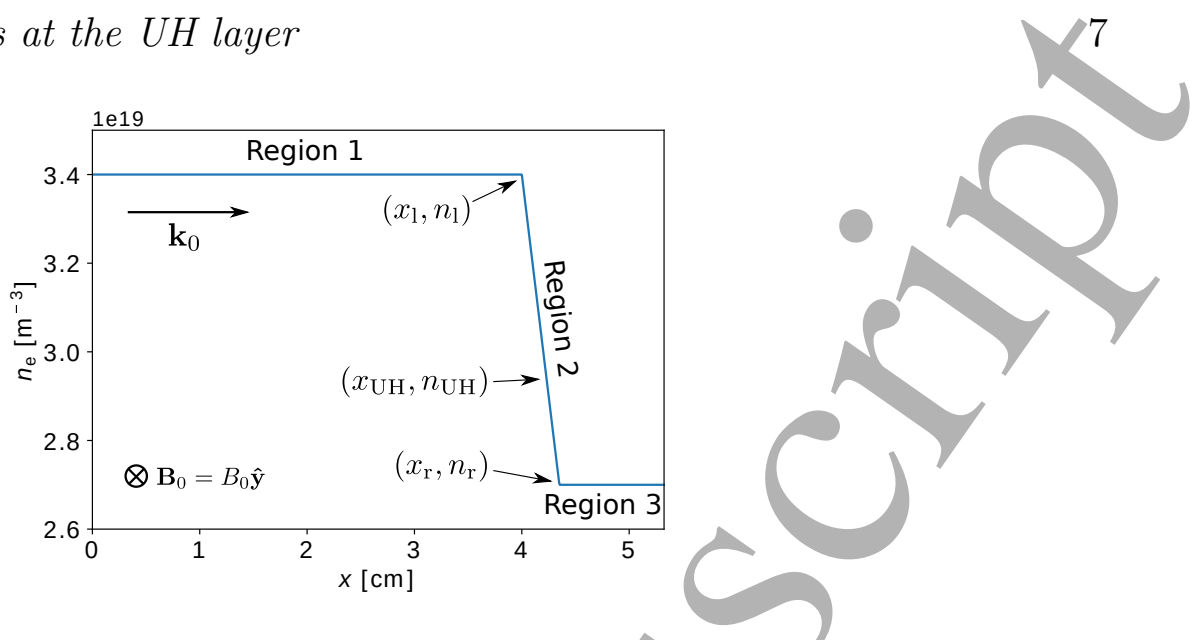

Figure 4. The geometry of the computational domain. All initial parameters except for the density are constant throughout the domain. The density profile is divided into three regions, two being constant (region 1 and 3 ) and one bridging the other two (region 2). Positions and densities mentioned in the text are shown in brackets. The pump, $\mathbf{k}_{0}$, is generated at the $x=0$ boundary and propagates right in the figure. The background magnetic field is constant and points perpendicularly to the direction of propagation.

is kept constant, an X-mode pump, $k_{0}$, is generated inside a plasma and is propagated to the UH layer by varying only the density with position. The two different approaches are illustrated in figure 3.

The computational domain is illustrated in figure 4 and can be split into 3 regions, denoted as shown in the figure. The plasma is a deuterium plasma with the initial electron density equal to the initial ion density, $n_{\mathrm{e}}=n_{\mathrm{i}}=n(x)$. The density varies with position and is described by

$$
n(x)= \begin{cases}n_{1} & x<x_{1} \\ n_{1}+\left(n_{\mathrm{r}}-n_{\mathrm{l}}\right) \frac{x-x_{1}}{x_{\mathrm{r}}-x_{\mathrm{l}}} & x_{1} \leq x<x_{\mathrm{r}}, \\ n_{\mathrm{r}} & x_{\mathrm{r}} \leq x\end{cases}
$$

where $n_{\mathrm{l}}=3.4 \times 10^{19} \mathrm{~m}^{-3}, n_{\mathrm{r}}=2.7 \times 10^{19} \mathrm{~m}^{-3}, x_{1}=4.00 \mathrm{~cm}, x_{\mathrm{r}}=4.35 \mathrm{~cm}$. The extend of the entire domain is $0.00 \mathrm{~cm} \leq x \leq 5.35 \mathrm{~cm}$ and there are a total of $n_{x}=6600$ grid points and $n_{\text {part }}=60000 n_{x}$ pseudoparticles of which half are electrons and the other half are deuterons. Regions 1 and 3 are constant for easier mode identification while region 2 bridges them. The initial background magnetic field and temperature are homogeneous and given by $B_{0}=B_{0 y}=3.35 \mathrm{~T}$ and $T_{\mathrm{e}}=T_{\mathrm{i}}=0.3 \mathrm{keV}$. The time step is chosen so that it is 0.95 multiplied the longest allowed by the Courant-Friedrichs-Lewy stability condition[30]. The output time step is $\delta t=\left(10.3 f_{0}\right)^{-1}=9.25 \times 10^{-13} \mathrm{~s}$ and the length of the simulations is around $50 \mathrm{~ns}$.

A pump wave at $f_{0}=105 \mathrm{GHz}$, denoted $\mathbf{k}_{0}$ in figure 4 , is generated at the $x=0.00$ $\mathrm{cm}$ boundary which propagates through region 1 and 2 until it reaches the UH layer at $x_{\mathrm{UH}}=4.25 \mathrm{~cm}$ and $n_{\mathrm{e}}=n_{\mathrm{i}}=2.91 \times 10^{19} \mathrm{~m}^{-3}$. The pump intensity is ramped up using an arctan-function with a characteristic time scale of $\tau_{\text {ramp }}=0.20 \mathrm{~ns}$ and 


\section{PIC simulations of PDIs at the UH layer}

the amplitude is varied over a number of simulations. The pump wave is generated in EPOCH by an oscillating pseudo-current at the boundary which, like an antenna, is able to excite an electromagnetic wave that can propagate into thedomain. The wave has an initial amplitude at the $x=0 \mathrm{~cm}$ boundary but is free to interact with the plasma throughout the domain. The pump wave is an X-mode wave which gets increasingly longitudinal near the UH layer. EPOCH can currently only excite transverse electromagnetic components which turns out to also excite an EBW wave at the same frequency. This unintended wave will be referred to either as the boundary wave or by $k_{\mathrm{B}}, f_{\mathrm{B}}$. The field boundary conditions are found by rewriting Maxwell's equations, using a current to excite waves at the $x=0.00 \mathrm{~cm}$ boundary and blocking incoming waves at the $x=5.35 \mathrm{~cm}$ boundary. The particles are initially thermally distributed and the particle boundary conditions at both boundaries replace any leaving particle with a new thermally distributed one. The thermally distributed particles give rise to thermally excited natural modes throughout the plasma.

Figure 5 shows the longitudinal component of the resulting electric field at two different points in time. The first shows the pump, $k_{0}$, ramping up and propagating through region 1 . The boundary wave, $k_{\mathrm{B}}$, can be seen near the $x=0.00 \mathrm{~cm}$ boundary. At the later time, the boundary wave has propagated further into region 1 , the pump has made it into region 2 and amplification is observed at the UH layer. Emerging from the UH layer, what appears to be the linearly converted wave, $k_{\mathrm{L}}$, is seen propagating back through region 1. A greater wavenumber and slower group velocity are contributing to a greater wave amplitude in order to conserve energy. While the pump only leads to relatively small density perturbations, the boundary and linearly converted wave, being EBWs, are carried by electron density perturbations[16] and reach relative amplitudes of $\max \left(n_{\mathrm{e}}(t) / n_{\mathrm{e}}(0)\right) \approx 1.2$ for $I_{\text {pump }}=17.4 \pm 0.5 \mathrm{MW} / \mathrm{m}^{2}$ and $\max \left(n_{\mathrm{e}}(t) / n_{\mathrm{e}}(0)\right) \approx 1.3$ for $I_{\text {pump }}=35 \pm 1 \mathrm{MW} / \mathrm{m}^{2}$. In figure 6 , the average longitudinal electron momentum is shown for the same time instances as in figure 5. Except for some minor differences, perhaps a small phase shift noticeable near the UH layer, the main features are the same as those observed in figure 5. The longitudinal waves in the electric field lead to acceleration of the electrons and thereby also to corresponding wave features in electron momentum. Lastly, figure 7 shows the electron density in time and space along with sketched out wayefronts of the waves excited at the boundary and the waves returning from the UH layer. The EBWs, which propagate as electron density perturbations, are clearly seen whereas the X-mode pump makes less of an impression on the background density. However, interference patterns are seen in the presence of multiple waves, e.g. above the $k_{\mathrm{B}}$-line where both the X-mode pump and the boundary wave propagate. Again, the same features are observed in this figure as the EBWs propagate as density perturbations which lead to longitudinal electric field which then cause longitudinal electron momentum to change. A plot similar to figure 7 but for the longitudinal electric field can be seen in figure 16.

An entirely inhomogeneous setup is also considered due to the possibility of lower threshold homogeneous PDIs in the homogeneous region 1 leading up to the UH layer. 


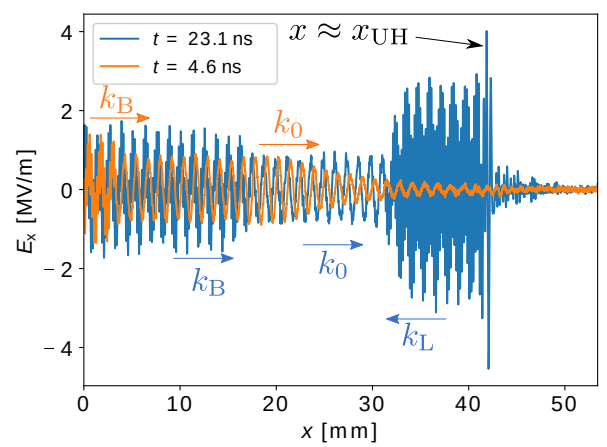

Figure 5. The longitudinal component of the electric field, $E_{\mathrm{x}}$, at two points in time as indicated on the legend. Colored labels refer to the waves present, and $x_{\mathrm{UH}}$ is the position of the UH layer. The pump intensity is $I_{\text {pump }}=17.4 \pm 0.5 \mathrm{MW} / \mathrm{m}^{2}$.

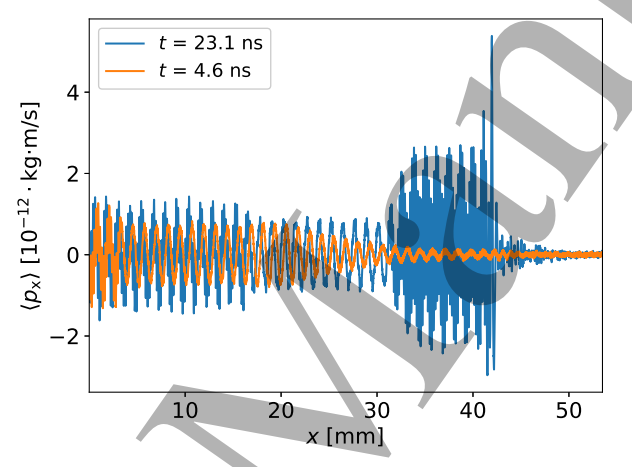

Figure 6. Averaged longitudinal electron momentum throughout the computational domain at the same points in time as shown in figure 5. The pump intensity is $I_{\text {pump }}=17.4 \pm 0.5 \mathrm{MW} / \mathrm{m}^{2}$.

While observed frequency lines in this setup will not be due to homogeneous PDI, $k$-analysis cannot be performed on the waves. The inhomogeneous setup has all the same physical parameters except the extend of the domain is $0.00 \mathrm{~cm}<x<1.35 \mathrm{~cm}$ with the density profile given again by equation (12) but for $n_{1}=5.4 \times 10^{19} \mathrm{~m}^{-3}$,

Figure 7. Electron density plotted in space and time. The dashed blue, green and black lines are the approximate wave fronts of the waves indicated. The vertical dashed and dotted lines mark $x_{1}$ and $x_{\mathrm{r}}$ as illustrated in figure 4 . The pump intensity is $I_{\text {pump }}=17.4 \pm 0.5 \mathrm{MW} / \mathrm{m}^{2}$. 
$n_{\mathrm{r}}=2.7 \times 10^{19} \mathrm{~m}^{-3}, x_{1}=0.00 \mathrm{~cm}, x_{\mathrm{r}}=1.35 \mathrm{~cm}$. This means that all parameters are exactly the same at the UH layer and the parameters at $x=1.00 \mathrm{~cm}$ correspond to the homogeneous region 1 for the setup illustrated in figure 4 .

\section{Results}

4.1. Group velocity found by tracking the returning wave front

Figure 8. The process of tracking the wave front of the returning EBWs. The blue line shows the absolute value of the longitudinal component of the electric field, $E_{x}$. The orange dots are the average of the blue line over $3 \mathrm{EBW}$ wavelengths according to theory. The green line is an arctan fit to the orange dots, the center of the fit is taken to be the position of the wave front. The pump intensity is $I_{\text {pump }}=21.2 \pm 0.9 \mathrm{MW} / \mathrm{m}^{2}$ and the time is $t=25.0 \mathrm{~ns}$.

To show that the wavefront labeled as $k_{\mathrm{L}}$ in figure 5 is indeed an EBW, the group velocity is estimated by tracking the position of the wavefront in time. The tracking is done by averaging $\left|E_{\mathrm{x}}\right|$ in space over $3 \mathrm{EBW}$ wavelengths and the fitting the function $y(x)=A_{0} \arctan \left(\left(x-x_{0}\right) / l\right)+y_{0}$ to it at particular point in time. The parameter $x_{0}$ is then taken to be the position of the wave front, see figure 8 . The group velocity should be constant throughout region 1 so doing this for a number of different times as the wave front propagates through region 1 should provide at linear relation between $x_{0}(t)$ and $t$, the slope of which is the group velocity, see figure 9 . Using this approach gives the group velocity $v_{\mathrm{g}, \mathrm{L}}=-(7.45 \pm 0.07) \times 10^{5} \mathrm{~m} / \mathrm{s}$ for the simulation shown in figure 5 . The group velocity can also be found from the dispersion relations as $v_{g}=\partial \omega / \partial k$. Solving the warm X-mode dispersion relation and the EBW dispersion relation numerically and calculating the group velocities at the pump frequency using the input parameters of the homogeneous region 1 as stated in section 3.2 yields $\left|v_{\mathrm{g}, 0, \text { theo }}\right|=6.92 \times 10^{6} \mathrm{~m} / \mathrm{s}$ and $\left|v_{\mathrm{g}, \mathrm{L} \text {,theo }}\right|=7.43 \times 10^{5} \mathrm{~m} / \mathrm{s}$ respectively. From this, it is seen that the returning wave is much too slow to be an X-mode wave. On the other hand, $v_{\mathrm{g}, \mathrm{L}, \text { theo }}$ is within the error of $v_{\mathrm{g}, \mathrm{L}}$ supporting the hypothesis that the returning waves are EBWs with a frequency around the pump frequency. 


\section{PIC simulations of PDIs at the UH layer}

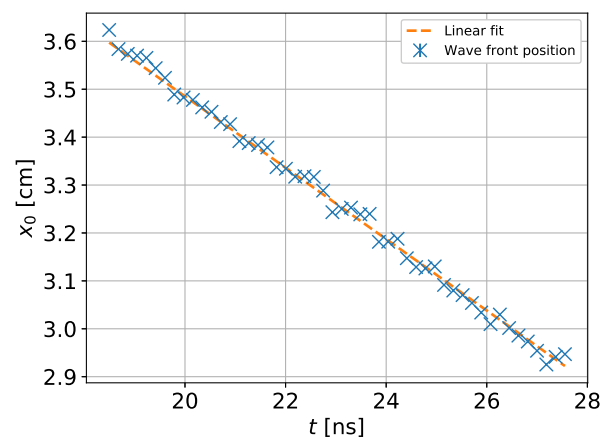

(1)

17

Figure 9. The tracked wave front position as a function of time (blue crosses) with a linear fit to give an estimate of the group velocity of the returning EBWs. The pump intensity is $I_{\text {pump }}=21.2 \pm 0.9 \mathrm{MW} / \mathrm{m}^{2}$.

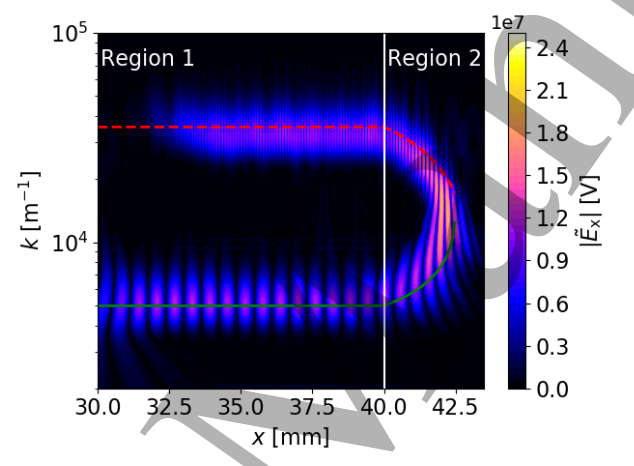

Figure 10. The absolute value of a CWT of the longitudinal component of the electric field, $E_{\mathrm{x}}$, near the UH layer at $t=22.5 \mathrm{~ns}$. The vertical solid white line marks the end of the first homogeneous region. The dashed red line and solid green line are numerical solutions to equations (11) for the linearly converted EBW and (7) for warm X-mode respectively. The Morlet wavelet is used as base function and the pump intensity is $I_{\text {pump }}=21.2 \pm 0.9 \mathrm{MW} / \mathrm{m}^{2}$.

\subsection{Spatial spectrum contains several peaks}

The origins of the returning EBWs is investigated by means of a continuous wavelet transform (CWT) in space using the Morlet wavelet as base function to look at the spatial spectrum as a function of position. Figure 10 shows the resulting spectrum when transforming the longitudinal component of the electric field after the pump has reached the UH layer. As the pump wave gets closer to the UH layer, $k_{0}$ increases by half an order of magnitude in good agreement with the warm X-mode dispersion relation. At the UH layer, the wave experiences amplification and comes to a halt. It is then seen that a wave starts propagating backwards, increasing the wavenumber in good agreement with the dispersion relation of a linearly converted EBW, confirming that X-B conversion has taken place.

To get a better resolution of the spectrum, the returning waves are transformed using a fast Fourier transform (FFT) in space as they propagate in region 1. Any FFT in this article will be using the orthonormal definition provided by the Python library 


\section{PIC simulations of PDIs at the UH layer}

Numpy. The absolute square of the resulting spectrum is shown in figure 11 along with a number of labels. The peak denoted by $k_{0}$ coincides the pump and the one denoted by $k_{\mathrm{L}}, k_{\mathrm{B}}$ is the combined peak associated with the linearly converted wave and the boundary wave. The four remaining labeled peaks, $k_{2}$ through $k_{5}$, are all shifted by an integer times $5.9 \pm 0.2 \mathrm{~mm}^{-1}$ relative to the $k_{\mathrm{L}}, k_{\mathrm{B}}$-peak. These peaks are likely due to PDIs as described in equations $(1 a-\mathrm{b}), k_{2}$ and $k_{3}$ being the downshifted and upshifted EBW respectively, as the downshifted EBW is typically the larger peak. While it is now seen that the returning waves consist of several EBWs, it does, however, appear that the pump is mainly converted into $k_{\mathrm{L}}$. All the above mentioned peaks are also observed when transforming the longitudinal electron momentum phase space.

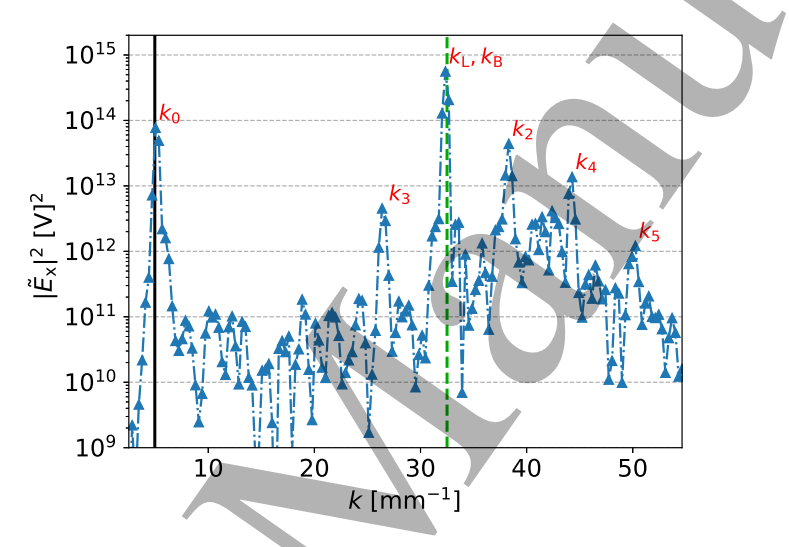

Figure 11. The square of the FFT of the longitudinal component of the electric field, $E_{\mathrm{x}}$, into $k$-space in the range of X-mode and EBW at the pump frequency (blue triangles connected by a dash-dotted line). The solid black line marks the numerical solution to warm X-mode at the pump frequency. The dashed green line marks the numerical solution to EBW at the pump frequency. Red labels mark peaks that are discussed in the text. The pump intensity is $I_{\text {pump }}=21.2 \pm 0.9 \mathrm{MW} / \mathrm{m}^{2}$ and the transform is over $2 \mathrm{~cm}<x<4 \mathrm{~cm}$ at $t=46.5 \mathrm{~ns}$.

\subsection{Spectral density shows scattering at shifted frequencies}

The spectral density of the returning waves is found using an FFT of time into frequency space and is compared to the experimentally observed peaks. Figure 12 shows the spectral density of $E_{\mathrm{x}}$ near the UH frequency in region 1 after the returning waves have reached jt. The main peak, labeled $f_{0}$, coincides with the pump frequency and has contributions from the pump wave, the linearly converted wave and the boundary wave. Found symmetrically around the main peak are the two smaller shifted peaks labeled $f_{2}$ and $f_{3}$. The shift in frequency of the peaks agrees well with the experimentally observed shifts. It is noted that approximating the shift by the LH frequency underestimates it while equation (2) overestimates it. This is in agreement with [23]. An additional peak, labeled $f_{4}$, is found at twice the shift of $f_{2}$. This peak could be a further PDI daughter and is likely to be the wave that leads to $k_{4}$ in figure 11. 


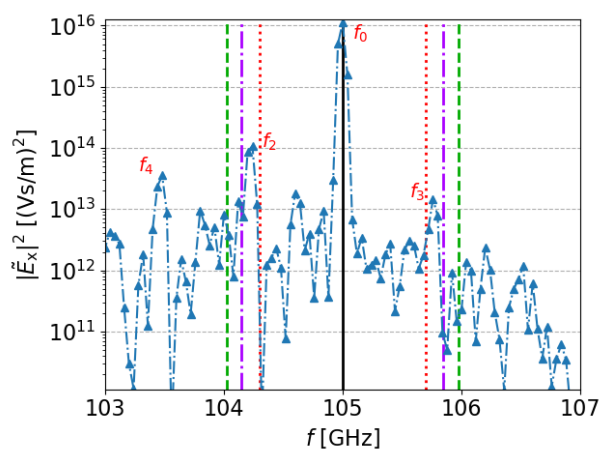

Figure 12. The spectral density of the longitudinal component of the electric field, $E_{\mathrm{x}}$, near the pump frequency (blue triangles connected by a dash-dotted line). The solid black line marks the pump frequency. The red dotted lines, the purple dashdotted lines and the green dashed lines are shifts corresponding to the LH frequency, experimental observations[23] and equation (2) respectively. The pump intensity is $I_{\text {pump }}=21.2 \pm 0.9 \mathrm{MW} / \mathrm{m}^{2}$ and the transform is over $20 \mathrm{~ns}<t<45 \mathrm{~ns}$ at $x=3.5$ $\mathrm{cm}$.

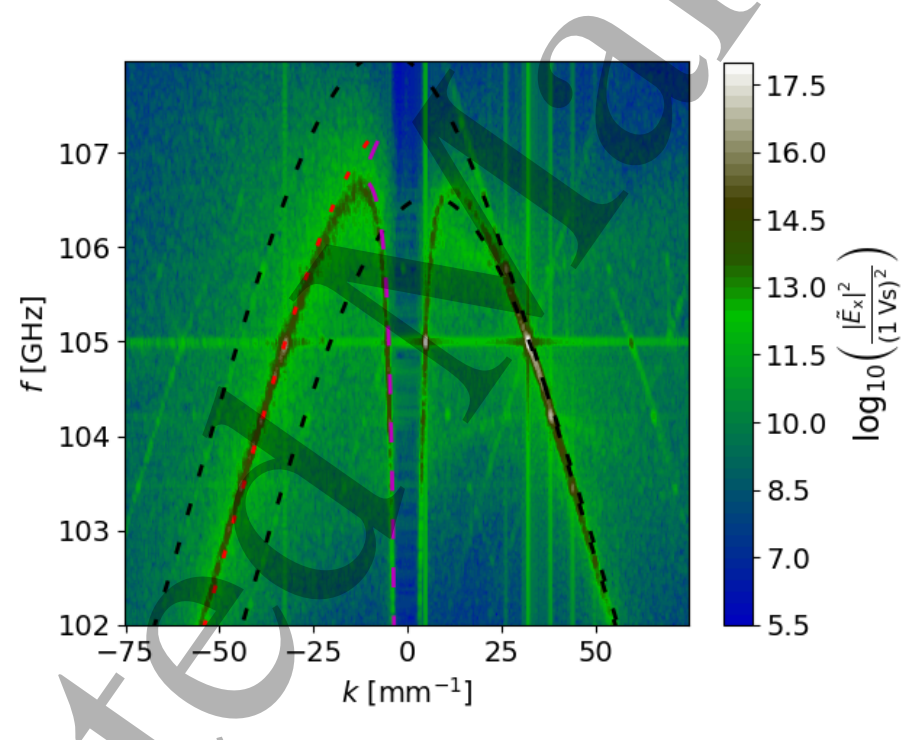

Figure 13. $f$-and $k$-space of the longitudinal component of the electric field, $E_{\mathrm{x}}$, near the pump frequency. The dashed purple line is the expected X-mode dispersion line and the dotted blue line is the EBW branch. The black dotted lines are EBW dispersion lines shifted by the observed LH daughter mode in figure 15. The pump intensity is $I_{\text {pump }}=21.2 \pm 0.9 \mathrm{MW} / \mathrm{m}^{2}$ and the transform is over $2 \mathrm{~cm}<x<4 \mathrm{~cm}$, $20 \mathrm{~ns}<t<46.5 \mathrm{~ns}$.

\subsection{Dispersion curves and relevant peaks along them}

To connect the peaks observed in the spectra shown in figures 11 and 12, the longitudinal component of the electric field is transformed in both $x$ and $t$ to give a spectrum in $k$ and $f$ using a 2D FFT. The absolute square of the resulting spectrum near the pump frequency is shown in figures 13 and 14. A number of lines and peaks are visible in this part of the spectrum. The warm X-mode and EBW dispersion relations from section 2 have been plotted in figure 13 for negative $k$ as a dashed purple line and red dotted 
PIC simulations of PDIs at the UH layer
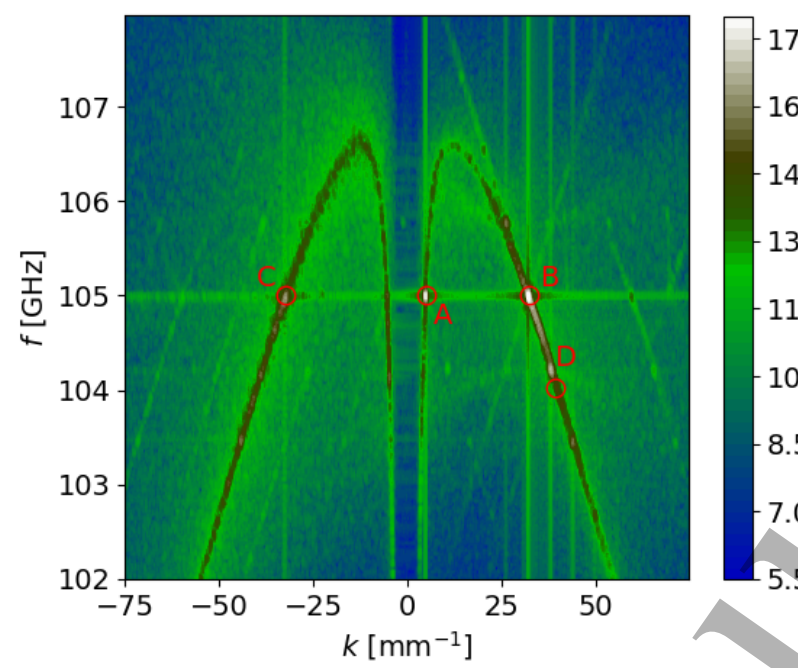

Figure 14. Same spectrum as shown in figure 13 without the dispersion lines but with major peaks labeled. Labels A through D mark selected peaks, A being the pump, $\mathrm{B}$ the linearly converted wave, C the boundary waye and D the downshifted EBW. The red circles denote theoretical predictions based on equations $(1 a),(2),(6),(7)$ and (11).

line respectively. Both lines fit well with dispersion lines present in the spectrum except where the observed lines meet. According to the dispersion relations, the lines do not meet, however, the EBW dispersion relation relies on a large $k$ approximation[28] which becomes doubtful in this point. The two lines are in fact expected to meet, as this is where linear conversion between X-mode and EBW can take place. The same lines are observed at the corresponding positive $k$-values. Additional lines are present and some will be addressed after the major peaks have been analyzed.

Figure 14 shows the same spectrum as figure 13 but the dispersion lines have been replaced by labels at the major peaks, and circles mark the analytical predictions presented in section 2 . Keeping in mind that $v_{g}=\partial \omega / \partial k$ and following the dispersion lines, a peak found along a line with a steep slope is a mode that propagates faster than one with at a less steep slope. Furthermore, a mode at a positive slope propagates in the positive $x$-direction while a negative slope indicates that the waves propagate in the negative $x$-direction. With this, the peak labeled $\mathrm{A}$ is seen to be a right propagating $\mathrm{X}$-mode at the pump frequency, i.e. the pump wave. $\mathrm{B}$ and $\mathrm{C}$ are both EBWs at the pump frequency. Based on the direction of propagation and peak value, $\mathrm{B}$ is the linearly converted EBW while $\mathrm{C}$ is the boundary EBW. Lastly, D is a downshifted EBW, which is seen to propagate left like the linearly converted EBW. The circles mark the predictions based on the relevant dispersion relations. In agreement with figure 12, the prediction for the downshifted EBW (D) overestimates the frequency shift but is found along the EBW dispersion line as expected. In addition to the marked peaks, a number of additional peaks are present, most notably some smaller peaks around the EBW dispersion line that $\mathrm{B}$ and $\mathrm{D}$ lie on, in particular $\left(k_{4}, f_{4}\right) \approx\left(45 \mathrm{~mm}^{-1}, 103.5 \mathrm{GHz}\right)$ 
as it is labeled in figures 11 and 12. It should be noted that all the above mentioned features are also present when transforming the electron density instead the longitudinal electric field component.

A PDI producing the downshifted EBW labeled D in figure 14 must also produce a low frequency daughter. The $f$ and $k$ spectrum near the LH frequency is shown in figure 15 and the expected position of the warm LH daughter wave, found in Bef [23], is marked by a red circle. As noted about the downshifted EBW, the overestimated prediction of the frequency shift means that the frequency of the warm LH daughter is also overestimated. The actual peak associated with the warm $\mathrm{LH}$ daughter is labeled E. Plotting the dispersion relation for the warm LH daughter, equation (5), gives the dashed magenta line which fits the observed dispersion line and the prediction is then seen to lie on the correct dispersion line. It is noted that the warm LH daughter must be back propagating like the downshifted EBW, judging from the slope of the dispersion line. A number of other smaller peaks can be seen, in particular at $\left(2 k_{1}, 2 f_{1}\right)$ and at $\left(3 k_{1}, 3 f_{1}\right)$. It is noted that the relative position of these three peaks resembles that of $\left(k_{L}, f_{L}\right),\left(k_{2}, f_{2}\right)$ and $\left(k_{4}, f_{4}\right)$ in figure 14. Shifting the EBW dispersion lines in figure 13 by adding and subtracting the observed warm LH daughter results in the two dotted black lines. The two shifted lines are seen to overlap for positive $k$, perhaps explaining why this EBW branch looks more smeared out between the peaks denoted B and D as is seen more clearly in figure 14 .

The absolute longitudinal component of the electric field is shown in figure 16 as a function of both position and time. The approximate wavefronts in region 1 of the relevant waves are indicated with lines and appropriate labels. Interference is observed when waves of comparable amplitude are found in the same space. The indicated white window in the figure is now used for FFTs. In these FFTs, the position in $(k, f)$ space of the pump wave, linearly converted EBW, downshifted EBW and warm LH daughter are determined as the local maximum near the expected location. To check if $(k, f)$ is conserved in the PDI and to see if the linearly converted EBW could act as an additional pump, two quantities are introduced as $\Delta_{\mathrm{PDI}, 0} \equiv\left(k_{1}+k_{2}-k_{0}, f_{1}+f_{2}-f_{0}\right)$ and $\Delta_{\text {PDI,L }} \equiv\left(k_{1}+k_{2}-k_{L}, f_{1}+f_{2}-f_{L}\right)$. A PDI can only take place if there is conservation of both $k$ and $f$, which means that a PDI converting the X-mode pump wave into the observed downshifted EBW and warm lower hybrid wave is only possible when $\Delta_{\mathrm{PDI}, 0}=0$. Likewise, it is possible for the linearly converted EBW to act as the pump, producing the same daughter waves, when $\Delta_{\mathrm{PDI}, \mathrm{L}}=0$. Recall that the wavenumbers depend on a number of parameters, including the density which varies throughout the numerical domain. While the frequency of a wave identified in the homogeneous region 1 is the same when the wave is at the UH layer, its wavenumber may have changed. It is expected that $\Delta_{\mathrm{PDI}, 0}$ is zero only at the UH layer so wavenumbers at the UH layer are calculated using the observed frequencies, the initial parameters at the UH layer and the relevant dispersion relations. The results are summarized in table 1 and the pump and the suspected PDI products show both frequency and wavenumber match at the $\mathrm{UH}$ layer, i.e. the error of $\Delta_{\mathrm{PDI}, 0}$ is greater than its value. In the homogeneous region 
PIC simulations of PDIs at the UH layer

\begin{tabular}{c|ll}
$(k, f)\left[\mathrm{mm}^{-1}, \mathrm{GHz}\right]$ & Observed in region 1 & Calculated at the UH layer \\
\hline Pump wave & $(4.4 \pm 0.3,105.00 \pm 0.05)$ & $(13 \pm 2,105.00 \pm 0.05)$ \\
Linearly converted EBW & $(31.7 \pm 0.3,105.00 \pm 0.05)$ & $(17.4 \pm 0.9,105.00 \pm 0.05)$ \\
Downshifted EBW & $(37.7 \pm 0.3,104.20 \pm 0.05)$ & $(26.8 \pm 0.5,104.20 \pm 0.05)$ \\
Warm LH daughter & $(-6.3 \pm 0.3,0.80 \pm 0.05)$ & $(-11 \pm 4,0.80 \pm 0.05)$ \\
\hline$\Delta_{\text {PDI }, 0}$ & $(27.0 \pm 0.9,0.0 \pm 0.2)$ & $(\mathbf{3} \pm \mathbf{7}, \mathbf{0 . 0} \pm \mathbf{0 . 2})$ \\
$\Delta_{\text {PDI }, \mathrm{L}}$ & $(-\mathbf{0 . 3} \pm \mathbf{0 . 9}, \mathbf{0 . 0} \pm \mathbf{0 . 2})$ & $\mathbf{( - 2} \pm \mathbf{5 , 0 . 0} \pm \mathbf{0 . 2})$
\end{tabular}

Table 1. Observed peaks in figures 14 and 15 found by locating the local maximum as well as the calculated quantities $\Delta_{\mathrm{PDI}, 0}$ and $\Delta_{\mathrm{PDI}, \mathrm{L}}$ introduced in the text. $k$-values are given in units of $\mathrm{mm}^{-1}$ and $f$ in GHz. The values at the UH layer are calculated using initial values at $x_{\mathrm{UH}}$ as stated in section 3.2. Bold numbers for $\Delta_{\mathrm{PDI}, 0}$ and $\Delta_{\mathrm{PDI}, \mathrm{L}}$ indicate conservation of both $k$ and $f$ within the error.

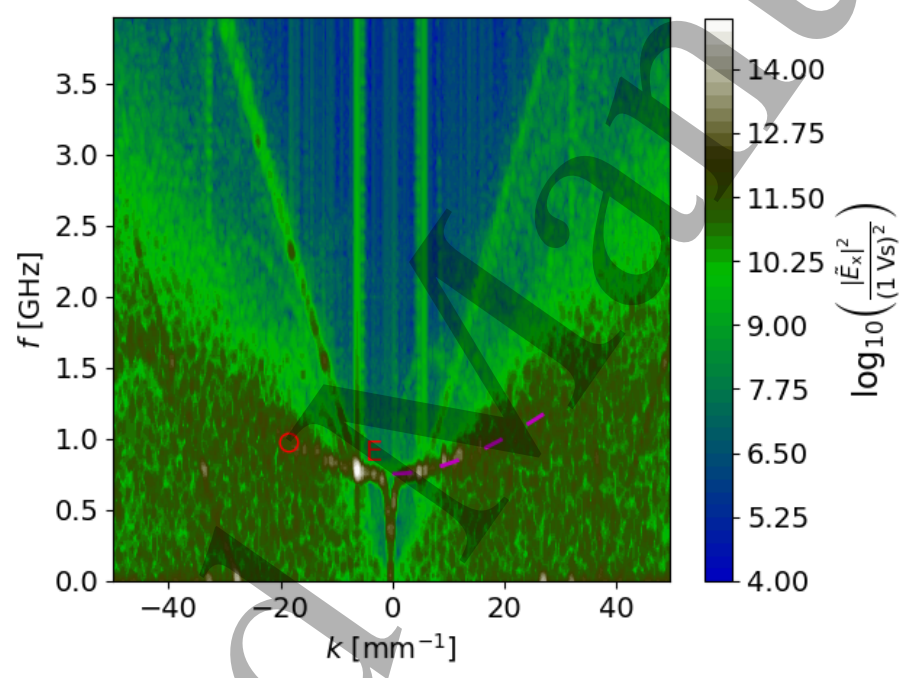

Figure 15. $f$ - and $k$-space of the longitudinal component of the electric field, $E_{\mathrm{x}}$, near the LH frequency. The label E marks the warm LH daughter while the red circle is the theoretical location found using equations (2) and (5). The magenta dashed line is the dispersion line of equation (5) for positive $k$. The pump intensity is $I_{\text {pump }}=21.2 \pm 0.9 \mathrm{MW} / \mathrm{m}^{2}$ and the transform is over $2 \mathrm{~cm}<x<4 \mathrm{~cm}, 20 \mathrm{~ns}<t<$ 46.5 ns.

1 , on the other hand, $\Delta_{\mathrm{PDI}, 0} \neq 0$ and this PDI is therefore only possible somewhere in region 2 near the UH layer where the selection rules of equations $(1 a)$ are exactly satisfied. Surprisingly, $\Delta_{\mathrm{PDI}, \mathrm{L}}=0$ within the error both at the UH layer and in the homogeneous region 1 . Theoretical thresholds and growth rates of this PDI are likely different from those of the PDI involving an X-mode pump wave. The implications of this are discussed in section 5 .

\subsection{Power of the daughter modes depends nonlinearly on pump intensity}

To investigate the relation between the intensity of the pump and of the PDI daughter wave, the pump intensity is varied over a number of simulations, keeping the simulation 
PIC simulations of PDIs at the UH layer

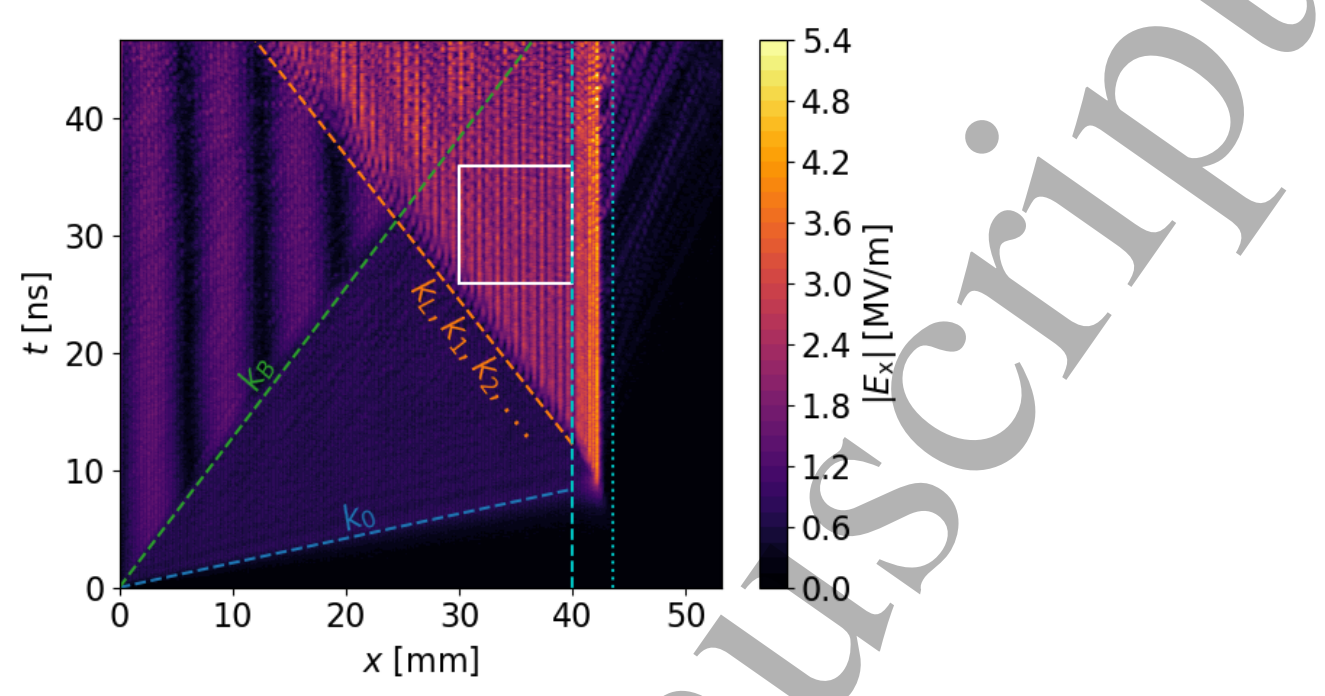

Figure 16. The absolute value of the longitudinal component of the electric field, $E_{\mathrm{x}}$, plotted in space and time. The dashed blue, green and orange lines are the approximate wavefronts of the waves indicated. The vertical dashed and dotted lines mark $x_{1}$ and $x_{\mathrm{r}}$ as illustrated in figure 4 . The white box enclosing $26 \mathrm{~ns}<t<36 \mathrm{~ns}$ and $30 \mathrm{~mm}$ $<x<40 \mathrm{~mm}$ marks the domain used for the Fourier analysis on which the power plot in figure 17 is based. The pump intensity is $I_{\text {pump }}=21.2 \pm 0.9 \mathrm{MW} / \mathrm{m}^{2}$.

geometry constant. For each simulation, the window indicated in figure 16 is used for FFTs into $(k, f)$-space and the spectral density is then found as the absolute square of the transformed fields. This window is chosen so that the wave composition and average parameters do not change significantly throughout. The window is placed close to the UH layer to minimize interactions occurring in the homogeneous region 1. Integrating the relevant peaks of the spectral density yields a quantity proportional to the power contained in the peak via Parseval's theorem. Some peaks are found to shift slightly as the pump intensity is increased so the peaks are integrated by first locating the local maximum of the spectral density inside a box of dimensions $5.7 \mathrm{~mm}^{-1}$ by 0.70 $\mathrm{GHz}$ near the expected position of the peak. Having located the maximum, another box of same dimensions is centered around it and the spectral density is numerically integrated inside this box. The error is estimated by calculating a mean level based on the spectrum around the edge of the box, including the first layer of points on either side of it. For a discussion of this shift, see section 5. The pump intensity is determined using the relations in chapter 5.4 of [31] with a cold approximation. The absolute squared fields are found by averaging the field components over an interval $2.3 \mathrm{~ns}<t<$ $3.7 \mathrm{~ns}$ at positions from $x=2 \mathrm{~mm}$ to $x=7 \mathrm{~mm}$ in $1 \mathrm{~mm}$ steps where the X-mode pump intensity should be far exceeding any other mode. A normalized power, $P_{\text {norm }}$, is introduced as the integrated peak values of the spectral density normalized to that of a low pump intensity setting above the PDI threshold, i.e. the normalization depends on what mode is being looked at and it provides a measure of how the power in that particular mode changes with the pump intensity. Based on [23], the inhomogeneous 


\section{PIC simulations of PDIs at the UH layer}

threshold is calculated to be $I_{\text {inhom }}=14.2 \mathrm{MW} / \mathrm{m}^{2}$. The $I_{\text {pump }}=17.4 \pm 0.5 \mathrm{MW} / \mathrm{m}^{2}$ simulation just above the inhomogeneous threshold is chosen as the normalization point since this is approximately the level that reaches the UH layer in [23].

Figure 17 shows the resulting plot of the normalized power of selected modes against the pump wave intensity scan. Above $I_{\text {inhom }}$ the power level of the PDI daughters, $\left(k_{1}, f_{1}\right)$ for the warm LH daughter and $\left(k_{2}, f_{2}\right)$ for the downshifted EBW, increase at a rate that looks to be more aggressive than linear dependence as suggested by the line proportional to $I_{\text {pump }}^{4}$. The curves of the daughter waves above $I_{\text {inhom }}$ follow each other closely, suggesting that the same nonlinear process is the main contributor to both waves. The daughter waves appear to increase nonlinearly with pump intensity until the last point where they start decreasing. The relative increase of power in the pump wave, $\left(k_{0}, f_{0}\right)$, and the linearly converted EBW, $\left(k_{\mathrm{L}}, f_{\mathrm{L}}\right)$, are seen to follow a linear dependence on pump intensity fairly well at lower pump intensities. At higher pump intensities, the pump and consequently the linearly converted EBW drop off slightly from the line indicating linear dependence. It is likely because new wave interactions start draining the pump as it is propagating to the UH layer which would cause the PDI daughter waves to drop off in the end. This is discussed in further in section 5. Around $I_{\text {inhom }}$, the daughter waves display a correlated fluctuating behavior but no obvious trend is seen. Below $I_{\text {inhom, }}$, the power level of the warm $\mathrm{LH}$ daughter fluctuates but stays at roughly the same level, showing that this is below the threshold and is dominated by a background level. This is supported by a noise test without a pump wave where only thermally excited waves and numerical noise contribute. The downshifted EBW follows an approximate linear dependence on the pump intensity. A similar analysis on the homogeneous region 1 before the returning waves return from the UH layer suggests a linear dependence on pump intensity throughout the X-mode and EBW branches. It appears that the pump wave also feeds energy into the branches through other means than the PDI of interest.

In the entirely inhomogeneous simulations, where the homogeneous region 1 has been replaced by a continuation of the linear density profile in region 2, there is no possibility of interfering homogeneous PDI daughter waves. The price is that modes cannot be separated in $k$. A measure of normalized power is found through an FFT of $E_{x}$ from time into $f$-space at the position $x=1.00 \mathrm{~cm}$ where the initial parameters are the same as is the homogeneous region 1 used in the preceding analysis. The FFT is over the interval $5 \mathrm{~ns}<t<15 \mathrm{~ns}$ and the absolute squared spectrum is numerically integrated over an interval of $0.70 \mathrm{GHz}$ centered around the frequencies corresponding to the experimentally observed shifts. If one or more local minima are found inside the interval, the integral is cut short, stopping at the minima. As with the homogeneous case, an error is estimated based on a mean level of the surrounding spectrum. Again, the first pump intensity level above the threshold is chosen as the normalization point, the resulting plot is shown in figure 18. The LH, $f_{1}$, and the downshifted EBW, $f_{2}$, daughter waves follow a similar nonlinear trend above the predicted threshold. Below the threshold, the LH daughter wave fluctuates with some large uncertainties on some points 
1

2

3

4

5

6

7

8

9
PIC simulations of PDIs at the UH layer

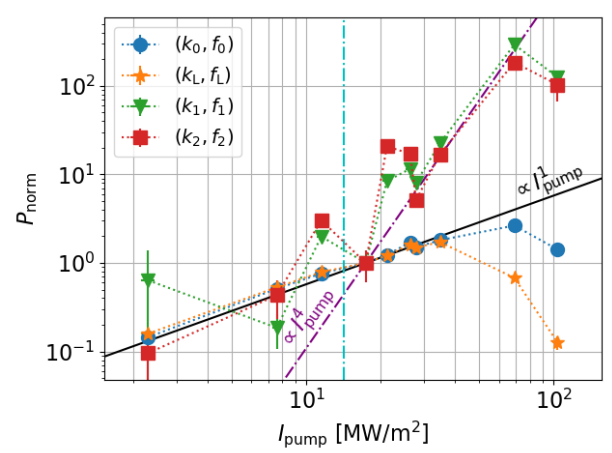

Figure 17. The normalized power contained in selected modes as the pump intensity is increased. The power is found by integrating peaks in the absolute squared $2 \mathrm{D}$ FFT of the electric field, $E_{\mathrm{x}}$, into $f$ - and $k$-space. The FFTs are over 26 $\mathrm{ns}<t<36 \mathrm{~ns}$ and $20 \mathrm{~mm}<x<30 \mathrm{~mm}$. The power is normalized so that $P_{\text {norm }}\left(I_{\text {pump }}=17.4 \pm 0.5 \mathrm{MW} / \mathrm{m}^{2}\right)=1$ at the lowest pump intensity simulation for all modes and the plot therefore does not show the relative power between modes. The black solid line is proportional to $I_{\text {pump }}$ and the purple dash-dotted line to $I_{\text {pump }}^{4}$. The theoretical inhomogeneous threshold is indicated by the vertical cyan dashed line.

while the downshifted EBW daughter wave displays a trend closer to linear dependence on the pump intensity. The figure is further discussed in the following section.

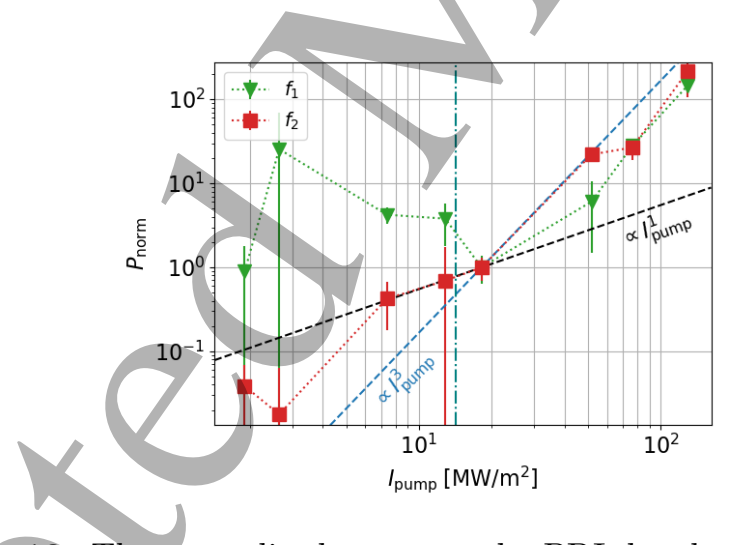

Figure 18. The normalized power at the PDI daughter frequencies, $f_{1}$ for the warm $\mathrm{LH}$ wave and $f_{2}$ for the downshifted EBW, in an entirely inhomogeneous density profile as the pump intensity is increased. The power is found by integrating the spectral density of the longitudinal component of the electric field, $E_{\mathrm{x}}$, around the PDI daughter frequencies. The FFTs are over $5 \mathrm{~ns}<t<15 \mathrm{~ns}$. The power of each PDI daughter is normalized to the power of the first point above the theoretically predicted PDI threshold. The plot therefore does not show the relative power between modes. The black solid line is proportional to $I_{\text {pump }}$ and the blue dash-dotted line

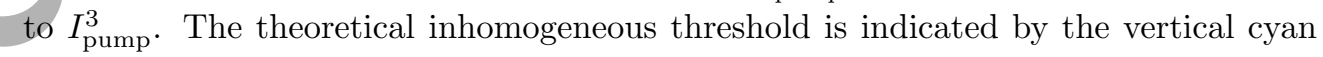
dashed line.

\section{Discussion}

As shown in section 4, the PIC code EPOCH was able to model nonlinear mode conversion through PDI under conditions similar to those of a magnetically confined 
fusion plasma. In particular, figure 12 displays a frequency spectrum with side peaks around the pump frequency, resembling that shown in [23]. Because of the homogeneous region 1 , the waves could be identified in both $f$ - and $k$-space, and it was confirmed that all the waves necessary for the PDIs described in section 2 were present. It was assumed that the decay takes place exactly at the UH layer as this is where the pump wave experiences the greatest amplification and the decay is therefore most likely to occur there. Figures 5 and 16 both show that $E_{x}$ is greatest at the UH layer, and table 1 confirms that the PDI conserves both wavenumber and frequency, i.e. $\Delta_{\text {PDI }, 0}=(0,0)$, at the UH layer. However, some of the dispersion relations are very sensitive around the UH layer and the error of the calculated wavenumbers therefore increases a lot. This means that $\Delta_{\mathrm{PDI}, 0}=(0,0)$ is found within the error at a range of positions around the UH layer. It was noted that the position of various peaks in $k$ - and $f$-space shift slightly with pump intensity. The decay may take place whenever the selection rules are satisfied so this shift can be explained by the pump reaching the PDI threshold further and further away from the UH layer as the intensity is increased. As the wavenumbers depend on position, the selection rules may then require a different frequency shift if the decay takes place at a different position due to the increase in pump intensity. This also explains why the linearly converted EBW appears to fall off quicker than the pump wave at higher pump intensities as less of the pump wave would then make it to the UH layer where the linear conversion takes place. It is also possible that the large amplitude density and temperature fluctuation carried by the EBWs in particular causes the position of the UH layer to shift around. Likewise, a portion of the injected energy may be deposited throughout the domain, causing the temperature to increase and the UH layer to shift. The electron temperature averaged over the entire domain at the end of the FFT window, i.e. at $t=36 \mathrm{~ns}$, is increased up to $6 \%$ within the error for a relatively high pump intensity simulation with $I_{\text {pump }}=69 \pm 2 \mathrm{MW} / \mathrm{m}^{2}$. The ion temperature increase is even smaller, $0.5 \%$ for the same parameters and they therefore represent fairly small changes to the parameters.

The theoretical predictions are based on a dipole approximation saying that $k_{0}=k_{1}+k_{2} \approx 0$, although [23] notes that this approximation is perhaps not particularly accurate and may be the reason why the predicted frequency shift overshoots as is seen in figures 12, 14 and 15. While table 1 shows that $\Delta_{\mathrm{PDI}}=(0,0)$ at the UH layer, it also shows that the dipole approximation does not appear to be valid according to the dispersion relations. This is confirmed by the CWT plotted in figure 10 where a dipole approximation would have been appropriate only in the homogeneous region 1 but the significant increase in wavenumber leading up the UH layer means that $k_{0}$ becomes comparable to that of the EBWs. Interestingly, table 1 also shows that another PDI would be possible, namely decay of the linearly converted EBW into the same downshifted EBW and warm LH wave as the other process. This process appears to satisfy both $k_{L}=k_{1}+k_{2}$ and $f_{L}=f_{1}+f_{2}$ within the error at the UH layer but also in the homogeneous region 1. Moving the FFT window shown in figure 16 along the propagating returning waves suggests that more of the PDI daughter waves are generated 


\section{PIC simulations of PDIs at the UH layer}

as they propagate through the homogeneous region 1 . This indicates that both the Xmode and the linearly converted EBW act as a pump for PDIs in the homogeneous simulations.

As seen in figure 17, the pump wave power near the UH layer is not exactly linear with the injected pump intensity. In particular the pump power is seen to drop off for the two highest pump intensity simulations which suggests that additional wave dynamics take place in the homogeneous region. As the pump intensity is increased, the spectra corresponding to figures 14 and 15 go from showing mainly just the expected dispersion lines and the peaks of interest in this article, to showing several additional peaks and lines. Some of these lines can easily be shown to conserve wave number and frequency in PDI processes involving natural modes and large peaks that are already present in the spectra. This includes mixing of the pump wave, Ainearly converted EBW and boundary EBW with the first several EBW bands and harmonies. The first harmonic EBW branches eventually get so smeared out that the notion of individual peaks are lost and even the pump and linearly converted wave drop off significantly. At this point, a stationary state is not reached at the end of the simulation and the description in section 2 is insufficient. The power of the all the waves of interest drop off, perhaps because a secondary PDI threshold has been reached.

The threshold of a homogeneous PDI is generally much lower than that of an inhomogeneous threshold, however, the growth rate is also expected to be smaller and placing the FFT window close to the UH layer therefore limits the effects of a homogeneous PDI of the linearly converted EBW. An analysis of the boundary wave in the part of the homogeneous region 1 that is never reached by the returning waves from the UH layer suggests that/an EBW may indeed decay into the observed daughter wave. The homogeneous threshold of this process is appears to be around the $34.7 \pm 0.1 \mathrm{MW} / \mathrm{m}^{2}$ simulation. By assuming that pump wave and boundary wave are the main contributions to the electric field for $3 \mathrm{~mm}<x<16 \mathrm{~mm}$ and $25 \mathrm{~ns}<t<27 \mathrm{~ns}$, i.e. in the leftmost region in figure 16, the threshold of this homogeneous PDI with an EBW as pump is found to be $3.6 \pm 0.5 \mathrm{MW} / \mathrm{m}^{2}$ in terms of the power of the EBW using results from $[31,26]$. As the boundary wave is the same as the linearly converted wave, differing only by the sign of $k$, the homogeneous threshold for the linearly converted EBW is the same. Furthermore, the threshold is the same in terms of the X-mode pump wave, i.e. the horizontal axis in figure 17, since the majority of the pump is converted linearly into EBW at the UH layer for this power level. For this reason, the observed modes have a contribution from both the inhomogeneous PDI of the pump wave at the UH layer and the homogeneous PDI of EBWs. This setup is good for mode identification within the homogeneous region, but the resulting power plot in figure 17 is therefore also affected by contributions from several processes. Because of that, a number of simulations with no homogeneous region at different pump intensities were run and peaks associated with PDI in $f$-space were still observed and these inhomogeneous simulations resulted in the power plot in figure 18. Modes at the pump frequency are omitted in the inhomogeneous power plot as they cannot be separated, but comparing 


\section{PIC simulations of PDIs at the UH layer}

figures 17 and 18, the latter is easier to read. In both plots, both PDI daughter modes increase nonlinearly with pump intensity although the homogeneous region appears to cause a more aggressive trend. In both plots, the warm LH mode fluctuates and displays no obvious trend below the theoretical threshold whereas the downshifted EBW appears to increase with pump intensity albeit slower than above the threshold. It is noted that the inhomogeneous simulations produced relatively high power levels for the warm LH daughter below the threshold. As seen in figure 15 general level of the spectrum near the LH frequency is close to the dispersion line of the LH waves, producing a less peaked spectrum which the sometimes large error bars testament. It should be added that frequency is not conserved in PDI for some of the simulations below the threshold if the frequency of the local maximum in the integration intervals is used. This suggests that some of the relatively large values in the power plot below the threshold are not from PDI. All in all, the entirely inhomogeneous simulations support that the observed inhomogeneous threshold agrees with the theoretical prediction of $14 \mathrm{MW} / \mathrm{m}^{2}$ within a margin of $5 \mathrm{MW} / \mathrm{m}^{2}$.

In the simulations, the X-mode pump has a significant longitudinal component on the incoming side of the UH layer but EPOCH can only excite the transverse components which is likely why both an X-mode and an EBW at the pump frequency are excited at the boundary. The amplitude of the EBW is comparable to the X-mode but propagates much slower. If the unintended EBW reaches the UH layer, it may introduce new dynamics and this sets a limit on the length of the simulation and, as a consequence, also a limit on the FFT frequency resolution. A better resolution would require a larger domain but the simulations are already quite expensive. Improving the resolution could potentially allow for identification of smaller peaks in the ion gyro frequency range associated with secondary PDI processes which are expected to be important for higher pump intensities. The 1D approximation may also be problematic considering that the $k$-selection rule in equation $(1 a)$ could have a preferred direction for the PDI daughter waves that might not be possible in $1 \mathrm{D}$. All of this could be the reason for the discrepancy between the observed frequency shifts in experiments and in the simulations of the present article, however, it may also just be due to experimental uncertainty in measured plasma parameters.

To extend the work presented in this article, different density gradient length scales could be compared and the effects of an inhomogeneous magnetic field and temperature profile should be taken into account as well. The location of the UH layer also depends on these parameters and a nonzero gradient length scale for any of the parameters therefore changes the width of the PDI interaction region and thus also affects the threshold. However, as mentioned earlier, an inhomogeneous magnetic field requires a $2 \mathrm{D}$ simulation which is outside the scope of this article.

Lastly, some waves appear to actually propagate past the UH layer. In figure 16, a weak wavefront beyond the $\mathrm{UH}$ layer in region 3 is seen to appear in the last $10 \mathrm{~ns}$ of the simulation. An FFT suggests that the wave propagating is a twice downshifted EBW with $(k, f)=\left(-k_{4}, f_{4}\right)$ where $k_{4}$ and $f_{4}$ are as seen in figures 11 and 12 . The wave 
can propagate because it is shifted below the UH frequency at any point of the domain. This is, however, highly dependent on the density profile and does not translate to the experiments in question.

\section{Conclusion}

For the first time, it was confirmed through mode identification that PIC codes are capable of modeling parametric decay of waves in the electron cyclotron range of an underdense magnetically confined fusion plasma by reproducing experimental spectra for ASDEX Upgrade relevant plasma parameters. Propagating an X-mode pump to its UH layer produced a spectrum in $f$ - and $k$-space with several modes which satisfied the selection rules necessary for PDI. It was confirmed that an X-mode pump can indeed be converted into a downshifted EBW and a warm LH wave near the UH layer. The frequency shift of the downshifted EBW was found to be similar to the observed shift, located between the theoretically predicted shift based on a dipole approximation and a simple LH frequency shift, which provided an upper and lower bound respectively as has previously been noted about experimental data. An upshifted peak corresponding to recombination of the warm LH daughter and the pump was also observed. Additionally, linear X-B conversion was observed and another PDI with the linearly converted EBW as pump was found to occur as well. The EBW pump was able to decay through PDI with a much lower threshold in the homogeneous region where the selection rules of the X-mode pump were not satisfied. The remaining modés displayed shifts in $f$ and $k$ that suggest that they were the products of either similar PDIs or further decay of the products of the PDI of interest. The power of the linearly converted EBW was seen to depend approximately linearly on the pump intensity, $I_{\text {pump }}$, whereas the power of the PDI daughter waves was seen to increase faster than linearly above a theoretically predicted threshold, approximately as $I_{\text {pump }}^{4}$ for simulations with a homogeneous region and as $I_{\text {pump }}^{3}$ for entirely inhomogeneous simulations. Below this threshold, the daughter waves depend differently on the pump intensity. This confirms that the suspected linearly converted EBW is created through a linear process whereas the suspected daughter waves are created in a nonlinear process and the threshold of this nonlinear process agrees with the theoretical prediction.

Following the results of this article, PIC codes can be used to study PDI in microwaye experiments in magnetically confined plasmas. For future experiments, it is favorable to plan shots to avoid PDI, which simulations like the ones presented here can assist in. One such microwave experiment is 2nd harmonic ECRH. According to [26], a pump wave in a nonmonotonic density profile may decay through a PDI, producing a pair of daughter waves trapped between two UH layers. With each passing at an UH layer, the daughter waves experience a situation similar to the pump wave described here. Another subject of interest is to investigate PDIs from an EBW pump wave. This is of interest in X-B and O-X-B heating schemes as EBWs may be used to heat overdense plasmas but could potentially distribute energy in a much less localized 


\section{PIC simulations of PDIs at the UH layer}

manner. Instabilities like the ones described in this article are expected to be important in future machines such as ITER.

\section{Acknowledgments}

This work has been supported by research grant 15483 from VILLUM FONDEN. This work was performed using resources provided by the ARCHER HPC through grant EP/R029148/1, the Marconi HPC, as well as by the Cambridge Service for Data Driven Discovery (CSD3) operated by the University of Cambridge Research Computing Service (http://www.csd3.cam.ac.uk/), provided by Dell EMC and Intel using Tier-2 funding from the Engineering and Physical Sciences Research Council (capital grant EP/P020259/1), and DiRAC funding from the Science and Technology Facilities Council (www.dirac.ac.uk).

\section{References}

[1] V P Silin 1965 Sov. Phys. JETP 211127

[2] Y M Aliev et al 1966 Sov. Phys. JETP 23626

[3] T Amano et al 1969 J. Phys. Soc. Japan 26529

[4] A D Piliya 1971 Proc. 10th Int. Conf. Phenomena in Ionized Gases (Oxford) ed R N Franklin (Oxford: Donald Parsons \& Co. Ltd) p 320

[5] M N Rosenbluth 1972 Phys. Rev. Lett. 29565

[6] A D Piliya 1973 ZhETF. Pis. Red. 17374

[7] Course of Theoretical Physics. Volume 1: Mechanics, 2nd edition, L D Landau and E M Lifshitz, Pergamon Press, Oxford (1969)

[8] J Falk et al 1978 Applied Physics Letters 322

[9] G A Gusev et al 1975 Radiophysics and Quantum Electronics 187

[10] W Seka et al 2009 Phys. Plasmas 16052701

[11] H W Hendel et al 1973 Physical Review Letters, 314

[12] F S McDermott et al 1982 The Physics of Fluids 251488

[13] M Porkolab and B I Cohen 1988 Nucl. Fusion 28239

[14] J Li et al 2001 Plasma Phys. Control. Fusion 431227

[15] A Surkov et al 2005 32nd EPS Conf. on Plasma Physics vol 29C (ECA) P-5.103

[16] Heinrich Peter Laqua 2007 Plasma Phys. Control. Fusion 49 R1

[17] W. A. Bongers et al 2012 EPJ Web Conf. 3203006

[18] S K Nielsen et al Experimental characterization of anomalous strong scattering of mm-waves in TEXTOR plasmas with rotating islands 2013 Plasmas Phys. Control. Fusion 55115003

[19] S. Kubo et al 2016 JINST 11 C06005

[20] S K Nielsen et al Recent development of collective Thomson scattering for magnetically confined fusion plasmas Phys. Scr. 92 (2017) 024001

[21] S K Hansen et al 2019 Phys. Plasmas, 26, 062102

[22] E Z Gusakov et al 2006 Plasma Phys. Control. Fusion 48 B443

[23] S K Hansen et al Parametric decay instabilitiy near the upper hybrid resonance in magnetically confined fusion plasmas Plasma Phys. Control. Fusion 59 (2017) 105006

[24] I Y Dodin et al 2017 Phys. Plasmas 24032119

[25] E. Z. Gusakov and A. Yu. Popov Low-Threshold Parametric Decay Instabilities in the Experiments on the Electron Cyclotron Resonance Heating in Tokamaks and Stellarators JETP Letters 91 (2010) 655 [Pisma Zh. Eksp. Teor. Fiz. 91 (2010) 724] 
[26] E Z Gusakov et al 2016 Physics of Plasmas 23082503

[27] A. V. Arefiev et al Kinetic simulations of X-B and O-X-B mode conversion and its deterioration at high input power 2017 Nucl. Fusion 57116024

[28] Plasma Physics: An Introduction, Richard Fitzpatrick, CRC Press, Taylor \& Francis Group (2015)

[29] T D Arber et al Contemporary particle-in-cell approach to laser-plasma modelling Plasma Phys. Control. Fusion 57 (2015) 113001

[30] Numerical Methods in Photonics, A. V. Lavrinenko, J. Læsgaards, N. Gregersen, F. Schmidt and T. Søndergaard, CRC Press (2015)

[31] Plasma Waves, 2nd Edition, Donald Gary Swanson, CRC Press (2003) 